\title{
Osseous Metaplasia Leading to Traction Retinal Detachment in the Nonphthisical Eye of Young Boy with Cytomegalovirus Retinitis
}

\author{
Gurunadh Atmaram Vemulakonda ${ }^{1 \#}$, Joshua A. Sonnen ${ }^{2}$ \\ ${ }^{1}$ Department of Ophthalmology, University of Washington, Seattle, USA; ${ }^{2}$ Department of Pathology, University of Washington, \\ Seattle, USA. \\ Email: "gatmav@gmail.com
}

Received April 10 ${ }^{\text {th }}, 2013$; revised May 10 ${ }^{\text {th }}, 2013$; accepted May 20 ${ }^{\text {th }}, 2013$

Copyright (C) 2013 Gurunadh Atmaram Vemulakonda, Joshua A. Sonnen. This is an open access article distributed under the Creative Commons Attribution License, which permits unrestricted use, distribution, and reproduction in any medium, provided the original work is properly cited.

\begin{abstract}
Title: Osseous Metaplasia Leading to Traction Retinal Detachment in the nonphthisical eye of Young Boy with Cytomegalovirus Retinitis. Purpose: To report a case of intraocular bone formation in an 11-year-old boy with a history cytomegalovirus (CMV) retinitis secondary to immunosuppression following Bone Marrow Transplantation due to hyper Immunoglobulin G (IgG) syndrome. Methods: An 11-year-old male presented with decreased vision in his better seeing eye. Clinical examination and Bscan ultrasound discovered a tractional retinal detachment with a large subretinal lesion. The patient was taken for surgical intervention. Results: The subretinal lesion was removed during surgery, and histopathologic evaluation revealed mature lamellar bone. Conclusion: This case shows that mature intraocular bone can be associated with tractional retinal detachment in a nonphthisical eye.
\end{abstract}

Keywords: CMV Retinitis; Retinal Detachment; Osseous Metaplasia

\section{Introduction}

While bone formation arising from metaplasia of the retinal pigment epithelium (RPE) has been reported in the literature, it remains a rare entity. It often occurs in eyes with multiple ocular surgeries, inflammation, severe trauma, long standing retinal detachment, or phthisis bulbi which have a poor visual prognosis [1-4]. The inflammation and anatomical disruption associated with these settings appear to cause the RPE to fibrose and rarely become bone. Because it is uncommon for children to suffer these ocular changes, most cases of subretinal bone formation occur in adults, but the age of presentation can be varied [1,2]. In addition, our case is unusual given the age of the patient, the relative sparing of vision until late in the disease process, as well as the inciting factor of cytomegalovirus (CMV) retinitis for the formation of bone and subsequent tractional retinal detachment.

\footnotetext{
*Supported in part by Research to Prevent Blindness (unrestricted grant to the University of Washington). We have no financial interest or gain relating to this research.

${ }^{\#}$ Corresponding author.
}

\section{Case}

An eleven-year-old boy presented to the University of Washington with decreased vision in his right eye. As an infant he was found to have hyper Immunoglobulin G (IgG) syndrome, which required bone marrow transplantation (BMT). At 1 year of age, he developed bilateral cytomegalovirus (CMV) retinitis as a result of the immunosuppression needed following BMT. He had remained immunosuppressed with hydrocortisone $5 \mathrm{mg}$ every day and intravenous immunoglobulin every 6 weeks. Ten years after his initial treatment for bilateral CMV retinitis, the patient presented with decreased vision in his formerly better seeing right eye. His visual acuity was 20/60 in his right eye and 20/400 in his left eye with intraocular pressures (IOP) of 7 in his right eye and 10 in his left eye. Slit lamp examination of the anterior chamber revealed a quiet eye with bilateral posterior subcapsular cataracts (PSCs). Dilated fundus examination of the right eye revealed vitreous cell, mild disc pallor, vascular attenuation, regressed CMV retinitis, but a new nasal elevated subretinal lesion with overlying fibrosis. No retinal detachment was seen. Dilated exam of 
the left eye revealed mild disc pallor, vascular attenuation, and regressed CMV retinitis that had involved the macula.

The patient returned a few months later with further decreased vision in his right eye. He was found to have $4 / 200$ visual acuity in his right eye and 20/125 in his left eye with IOPs of 9 in his right eye and 9 in his left eye. Anterior chamber exam revealed a quiet eye with a worsened $3+$ predominately nasal PSC in his right eye with a stable mild PSC in his left eye. Dilated exam of the right eye revealed a hazy view from dense vitreous cell along with the PSC. Through these hazy media, however the nasal elevated subretinal lesion appeared to have increased in size. In addition there was now a retinal detachment overlying the lesion along with a tractional retinal detachment with both pre-retinal and subretinal proliferative vitreoretinopathy extending from the 1 o'clock hour to the $10 o^{\prime}$ clock hour. The left eye appeared stable.

An ultrasound of the right eye was obtained which revealed a broad, thick highly reflective lesion extending into the mid vitreous nasally. No details were visible posterior to the lesion due to shadowing (Figure 1).

After discussion with the family, it was decided to proceed with surgical intervention. The patient underwent 20 gauge pars plana vitrectomy, pars plana lensectomy, preretinal membrane peeling, subretinal membrane peeling, excisional biopsy, 360 degree retinectomy, pefluorooctane assisted drainage of subretinal fluid, endolaser, and silicone oil tamponade of his right eye. At time of surgery, the large lesion was found to beneath the neurosensory. Indeed, when it was removed from the eye, the inner aspect that was just beneath the retina was white, while the outer aspect was darkly pigmented and appeared to be associated with the retinal pigment epithelium. Unfortunately the retina and RPE detached from the lesion when it was removed from the eye.

Pathology of the lesion revealed (Figure 2):

Gross description: An ovoid, hard tissue piece $1.5 \times 1$ $\times 0.4 \mathrm{~cm}$ thick that is near black on one surface and pearly white on the opposite surface. The white surface is lobulated and has the appearance of bone.

\section{Discussion}

While calcification can be seen in degenerating tissues, [1] ossification generally requires abundant blood supply [1] and a fibroblastic response [2]. The anatomy of the posterior segment including the RPE and choroid provided such a blood supply [5], while scarring and inflammation from the patient's previous CMV chorioretinitis provided the fibrovascular response needed. The inflammation and anatomical disruption associated with these settings appear to cause the RPE to fibrose and

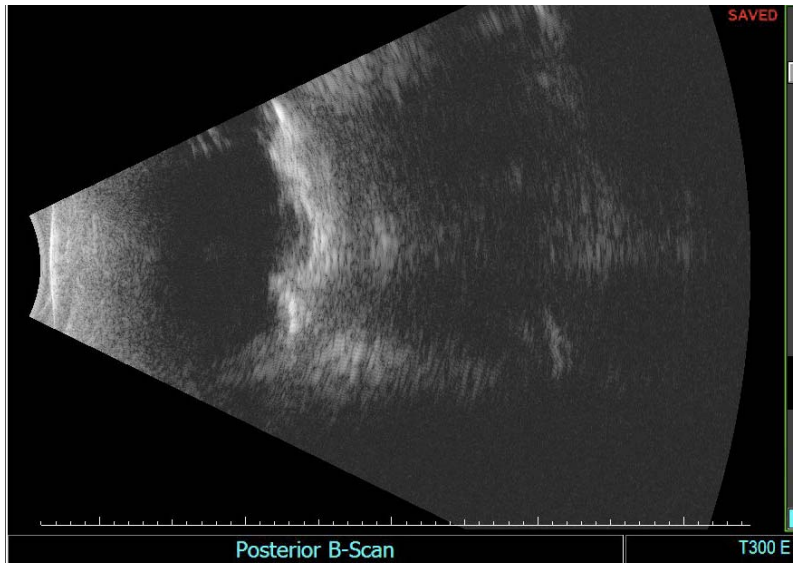

Figure 1. Bscan ultrasound imaging of the right eye was obtained which revealed a broad, thick highly reflective lesion extending into the mid vitreous cavity nasally. No details were visible posterior to the lesion due to shadowing.

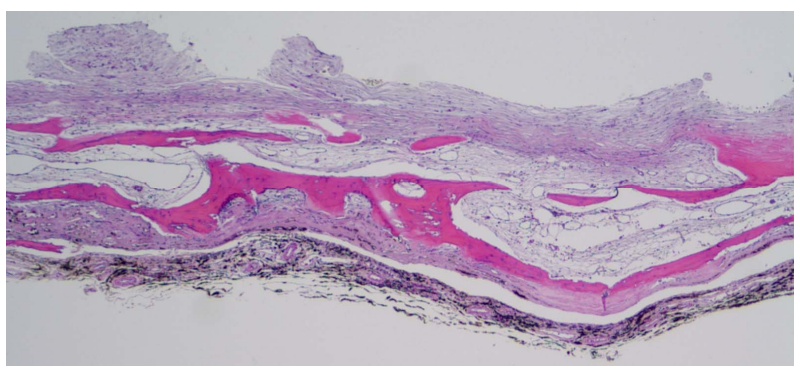

Figure 2. Microscopic description: a section of the resected subretinal membrane stained with hematoxylin and eosin reveal a layer of pigment laden choroid with underlying lamina of mature bony spicules with fibrotic marrow space and overlying fibrous tissue (200 $\times$ magnification).

rarely become bone. Boniuk et al found in their series that most ocular bone formation takes 10 - 12 years following insult [2]. This corresponds to our patient, whose insult occurred during infancy followed by the development of bone ten years later. Nonetheless, this range can be much larger anywhere from 10weeks [1] to 78 years [2]. Another interesting aspect of this case is that the patient has remained relatively immunosuppressed after his bone marrow transplant, but this did seem to prevent either the subsequent fibrosis or metaplasia. Finally, while serous retinal detachment associated with ocular calcification has been reported [3], this may be the first reported case of tractional retinal detachment associated with subretinal rather than pre-retinal bone formation in the absence of trauma or phthisis $[4,6]$.

\section{Conclusion}

This was a difficult case to manage, given the child's young age. In addition, this eye had initially been the better seeing eye, which made the surgical and medical 
management more difficult. If the patient had developed a serous retinal detachment, it could possibly have been managed medically with topical, intravitreal, systemic, or a combination of these treatments. Unfortunately, the tractional retinal detachment required more invasive treatment. As a result, prior to any surgical intervention, the patient was referred to two other retinal surgeons for their opinion on best management options. All retinal specialists were in agreement, that although the patient's visual prognosis was poor regardless of management strategy implemented, the patients worsening tractional retinal detachment could only be managed surgically. This provided tissue which confirmed the nonmalignant nature of the subretinal bone, while also allowing an attempt to reattach the retina. Unfortunately, despite heroic attempts, the patient's vision continued to worsen despite surgical reattachment, and he could only see the light with this eye 12 months following surgery.

\section{Acknowledgements}

Many thanks to Robert Kalina for his help with the management of this patient as well as this paper.

\section{REFERENCES}

[1] W. S. Duke-Elder, “Textbook of Ophthalmology,” St. Louis, Mosby, 1941, v. 3.
[2] E. M. Finklestein and M. Boniuk, "Intraocular Ossification and Hematopoiesis," American Journal of Ophthalmology, Vol. 68, No. 4, 1969, pp. 683-690.

[3] J. H. Song, J. H. Bae, M. I. Rho and S. C. Lee, "Intravitreal Bevacizumab in the Management of Subretinal Fluid Associated with Choroidal Osteoma,” Retina, Vol. 30, No. 6, 2010, pp. 945-951. doi:10.1097/IAE.0b013e3181c720ca

[4] Y. D. Yoon, T. M. Aaberg, T. H. Wojno and H. E. Grossniklaus, "Osseous Metaplasia in Proliferative Vitreoretinopathy,” American Journal of Ophthalmology, Vol. 125, No. 4, 1998, pp. 558-559. doi:10.1016/S0002-9394(99)80201-3

[5] J. M. Rhorbach, E. Liesenhoff and K. P. Steuhl, "Principles of Ocular Ossification Exemplified by Secondary Choroid Ossification,” Klinische Monatsblätter für Augenheilkunde, Vol. 197, No. 5, 1990, pp. 397-403.

[6] J. I. Loewenstein, R. N. Hogan and F. A. Jakobiec, “Osseous Metaplasia in a Preretinal Membrane," Archives of Ophthalmology, Vol. 115, No. 1, 1997, pp. 117-119. doi:10.1001/archopht.1997.01100150119023 